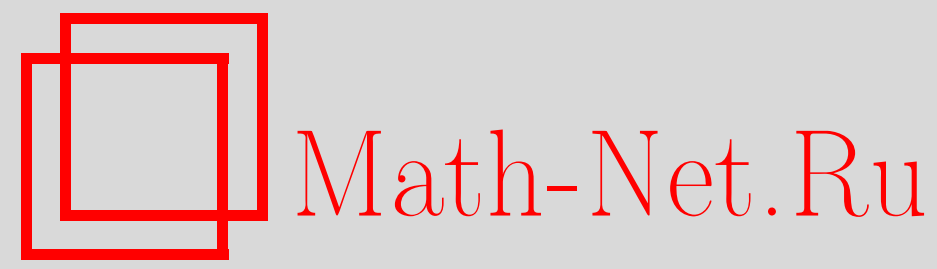

А. В. Степанов, Экстремальные порядковые статистики при изменении отношения порядка, Теория вероятн. и ее примен., 1996, том 41, выпуск 4, 896-900

DOI: https://doi.org/10.4213/tvp3243

Использование Общероссийского математического портала Math-Net.Ru подразумевает, что вы прочитали и согласны с пользовательским соглашением http://www . mathnet.ru/rus/agreement

Параметры загрузки:

IP: 52.23 .180 .231

26 апреля 2023 г., 08:45:26

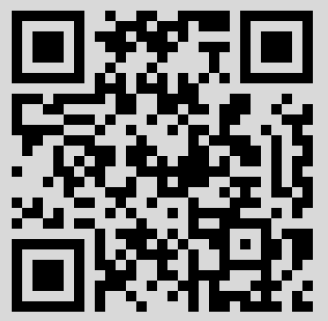


7. Kramkov D. O. Optional decomposition of supermartingales and hedging contingent claims in incomplete security markets. - Probab. Theory Relat. Fields, 1996, v. 105, p. $459-479$.

8. Kabanov Yu. M., Kramkov D. O. Asymptotic arbitrage in large financial markets. Finance and Stochastics (to appear).

Поступила в редакцию 18. VII. 1996

(C) $1996 \mathrm{r}$.

СТЕПАНОВ А. В.*

\section{ЭКСТРЕМАЛЬНЫЕ ПОРЯДКОВЫЕ СТАТИСТИКИ ПРРИ ИЗМЕНЕНИИ ОТНОШЕНИЯ ПОРЯДКА}

Пусть $X_{1}, X_{2}, \ldots$ - независимые случайные величины с обшей непрерывной функцией распределения $F(x)$. Фиксируем произвольную точку а на носителе вероятностной меры. Будем рассматривать последовательности случайных величин, состоящие из тех величин $X_{i}$, которые в той или иной степени стремятся к точке $a$. Кроме того, будем изучать индексы зтих случайных величин. По подходам и методам решений данная проблематика напоминает теорию рекордов.

Ключевые слова и фразы: рекордные моменты и величины, экстремальные порядковые статистики, последовательности случайных величин, приближающиеся к заданной точке.

1. Введение. Пусть здесь и везде далее $X_{1}, X_{2}, \ldots$ - независимые случайные величины (с.в.) с общей непрерывной функцией распределения $F(x)$. Пусть интервал $(\gamma, \beta)$ - носитель вероятностной меры, где $\gamma$ может быть равным $-\infty$, а $\beta$ может принимать значение $+\infty$. Рассмотрим с.в. $X_{1}, X_{2}, \ldots, X_{n}$ в неубывающем порядке: $\gamma<X_{1, n}<X_{2, n}<\cdots<X_{n, n}<\beta$. Величины $X_{r, n}(r \leqslant n)$ называют порядковыми статистиками. «Согнем» интервал $(\gamma, \beta)$ в окружность, соединив точки $\gamma$ и $\beta$. При такой постановке вопроса большей является та случайная величина, которая находится далее при обходе окружности вдоль часовой стрелки с началом обхода в точке $\gamma$ и концом обхода в точке $\beta$. Выберем теперь на окружности некоторую точку $a$ и зафиксируем ее. Начало обхода сдвинем в эту точку, а направление обхода сохраним. В этом случае вместо $\gamma$ мы имеем $a+0$, а вместо $\beta$ необходимо брать $a-0$. Отношение порядка среди с.в. $X_{i}$ изменится. Так, например, если с.в. располагались следующим образом:

$$
\gamma<X_{1, n}<X_{2, n}<\cdots<X_{n-2, n}<a<X_{n-1, n}<X_{n, n}<\beta,
$$

и если $\widetilde{X}_{r, n}$ - новые порядковые статистики, т.е.

$$
X_{n-1, n}=\widetilde{X}_{1, n}, \quad X_{n, n}=\widetilde{X}_{2, n}, \quad X_{1, n}=\widetilde{X}_{3, n}, \quad X_{n-2, n}=\widetilde{X}_{n, n},
$$

то $a+0 \prec \tilde{X}_{1, n} \prec \tilde{X}_{2, n} \prec \beta=\gamma \prec \tilde{X}_{3, n} \prec \cdots \prec \tilde{X}_{n, n} \prec a-0$. Здесь знаком $\prec$ обозначаем новое отношение порядка (см. также рис. 1).

Рассмотрим построенные определенным образом и связанные с экстремальными порядковыми статистиками последовательности с.в.: последовательности величин односторонних последовательных приближений («о.п.п.») $V_{(n)}^{a}$, моментов «о.п.п.»

*Калининградский государственный технический университет, Советский проспект, 1, 236000 Калининград, Россия. 
$T^{a}(n)$, а также последовательности $a$-круговых рекордных величин $X_{(n)}^{a}$ и $a$-круговых рекордных моментов $L^{a}(n)$ (данные понятия предлагаются нами впервые). Пусть

$$
\begin{aligned}
T^{a}(0) & =0, \quad X_{0}=V_{(0)}^{a}=\gamma, \\
T^{a}(n+1) & =\min \left\{j: j>T^{a}(n), X_{j} \succ X_{T^{a}(n)}\right\} \\
V_{(n)}^{a} & =X_{T^{a}(n)} \quad(n=0,1,2, \ldots) .
\end{aligned}
$$

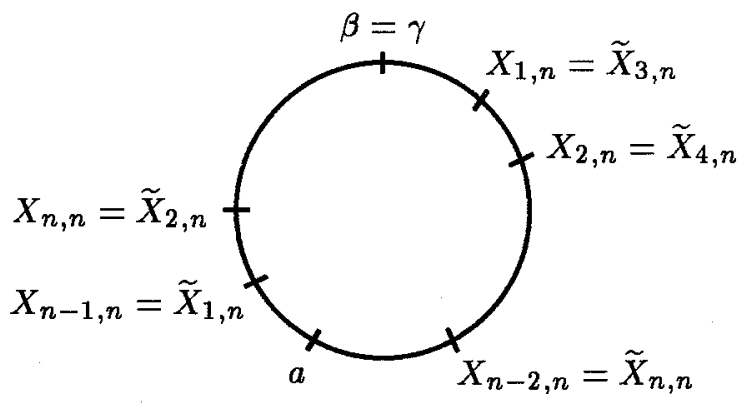

Pис. 1

Последовательность с.в. $V_{(n)}^{a}(n=0,1,2, \ldots)$ - это те из величин $X_{i}$, которые попадают в интервал $(\gamma, a)$ и последовательно приближаются к точке $a$ по часовой стрелке. Моменты $T^{a}(n)$ - индексы этих с.в. Подобные с.в. могут появляться в теории рекордов, в задачах, связанных с прочностью материала; автору приходилось сталкиваться с ними в некоторых прикладных задачах теории управления. Отметим, что последовательности $T^{a}(n)$ и $V_{(n)}^{a}$ существуют с вероятностью 1 и при $a \rightarrow \beta$ превращаются в последовательности рекордных моментов и величин соответственно. Далее, пусть

$$
\begin{aligned}
L^{a}(1) & =1 \\
L^{a}(n+1) & =\min \left\{j: j>L^{a}(n), X_{j} \succ X_{L^{a}(n)}\right\} \quad(n=1,2, \ldots) \\
X_{(n)}^{a} & =X_{L^{a}(n)} .
\end{aligned}
$$

Фактически величины $L^{a}(n)$ и $X_{(n)}^{a}$ являются рекордными моментами и величинами, при условии, что вместо отношения порядка < берется отношение ঝ, т.е. вместо $\beta$ выступает $a-0$. Отметим, что последовательности этих величин также реализуются с вероятностью 1 и при $a \rightarrow \beta$ величины превращаются в обычные рекорды.

Следующий пункт будет посвящен $a$-круговым рекордам. Мы увидим, что свойства $a$-круговых рекордных моментов полностью совпадают со свойствами рекордных моментов, однако поведение $a$-круговых рекордных величин отличается от поведения рекордных величин.

2. a-круговые рекордные моменты и величины. Приведем здесь подход к исследованию $a$-круговых рекордов, синтезируюшей в себе как подходы автора работы, так и методы, предложенные рецензентом статьи.

«Склейку» прямой в окружность можно рассматривать как формальное «подклеивание» интервала $(a, \beta)$ перед интервалом $[\gamma, a]$. Если же $-\infty<\gamma<\beta<\infty$, то 
можно рассмотреть с.в. $Y_{i}$ :

$$
Y_{i}= \begin{cases}X_{i}, & X_{i} \in[\gamma, a], \\ X_{i}+\gamma-\beta, & X_{i} \notin[\gamma, a] .\end{cases}
$$

Тогда $a$-круговые рекордные величины для $X$-ов можно выразить через классические рекордные величины для $Y$-ов:

$$
X_{(n)}^{a}= \begin{cases}Y_{(n)}, & Y_{(n)} \in[\gamma, a] \\ Y_{(n)}+\beta-\gamma, & Y_{(n)} \notin[\gamma, a]\end{cases}
$$

Отсюда получаем следующие результаты:

1) $L^{a}(n)=L(n)$

2) распределения самих $a$-круговых рекордных величин имеют вид

$$
\begin{array}{ll}
\mathbf{P}\left\{a<X_{(n)}^{a}<x\right\}=\frac{1}{(n-1) !} \int_{0}^{-\log (1+F(a)-F(x))} \nu^{n-1} e^{-\nu} d \nu & (a<x), \\
\mathbf{P}\left\{\gamma<X_{(n)}^{a}<x\right\}=\frac{1}{(n-1) !} \int_{-\log F(a)}^{-\log (F(a)-F(x))} \nu^{n-1} e^{-\nu} d \nu & (x<a) .
\end{array}
$$

Приведем здесь также результат, который вытекает из вышеизложенных рассуждений и, по мнению автора, представляет самостоятельный интерес в теории порядковых статистик.

Лемма 2.1. ПІусть $\operatorname{rank}_{a} X_{n}$ есть а-круговой ранг с.в. $X_{n}$ среди веяичия $X_{1}$, $X_{2}, \ldots, X_{n}$ (т.е. место расположения с.в. $X_{n}$ среди $X_{1}, X_{2}, \ldots, X_{n}$ с учетом нового

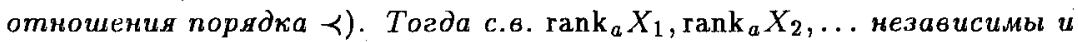

$$
\mathbf{P}\left\{\operatorname{rank}_{a} X_{n}=k\right\}=\frac{1}{n} .
$$

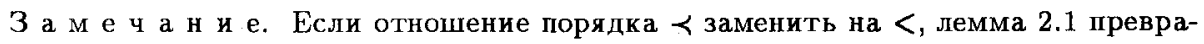
тится в известный результат Реньи (см., например, [2]).

В заключение отметим, что данные результаты остаются верными и без предположения $-\infty<\gamma<\beta<\infty$.

3. Моменты и величины «односторонних последовательных приближений». С сожалением отметим, что, в отличие от а-круговых рекордных моментов, функция распределения моментов «о.п.п.» $T^{a}(n)$ зависит от вида непрерывной функции распределения $F(x)$.

Утверждение 3.1. Пусть $j \geqslant 1$ справедлива формула

$$
\mathbf{P}\left\{T^{a}(1)=j\right\}=(1-F(a))^{j-1} F(a) .
$$

Д ок а з а т ель с тв о. Очевидно, что

$$
\mathbf{P}\left\{T^{a}(1)=j\right\}=\mathbf{P}\left\{X_{1}>a, \ldots, X_{j-1}>a, X_{j}<a\right\}=(1-F(a))^{j-1} F(a) .
$$

Из утверждения 3.1 вытекает, что $\mathbf{E} T^{a}(1)=\sum_{m=1}^{\infty} m F(a)(1-F(a))^{m-1}=1 / F(a)$.

Утверждение 3.2. Обобщением формулы (3.1) является бормула $\left(1 \leqslant j_{1}<\right.$ $\left.j_{2}<\cdots<j_{n}\right)(n \geqslant 1)$ :

$$
\begin{gathered}
\mathbf{P}\left\{T^{a}(1)=j_{1}, T^{a}(2)=j_{2}, \ldots, T^{a}(n-1)=j_{n-1}, T^{a}(n) \geqslant j_{n}\right\} \\
=\sum_{k=1}^{n} \frac{(1-F(a))^{j_{k}-1}}{\left(j_{n}-j_{k}\right) \cdots\left(j_{k+1}-j_{k}\right)\left(j_{k-1}-j_{k}\right) \cdots\left(j_{1}-j_{k}\right)} .
\end{gathered}
$$


З а м е ч а н и е. Крайние члены последней суммы выглядят следуюшим обра30M:

$$
\begin{array}{ll}
\frac{(1-F(a))^{j_{1}-1}}{\left(j_{n}-j_{1}\right) \cdots\left(j_{k}-j_{1}\right) \cdots\left(j_{2}-j_{1}\right)} & (k=1), \\
\frac{(1-F(a))^{j_{n}-1}}{\left(j_{n-1}-j_{n}\right) \cdots\left(j_{k}-j_{n}\right) \cdots\left(j_{1}-j_{n}\right)} & (k=n) .
\end{array}
$$

Д ок а за т е льс тв о у т в е жд д н и

$$
\begin{aligned}
& \mathbf{P}\left\{T^{a}(1)=j_{1}, T^{a}(2)=j_{2}, \ldots, T^{a}(n-1)=j_{n-1}, T^{a}(n) \geqslant j_{n}\right\} \\
& =\mathbf{P}\left\{\bigcap_{1 \leqslant i \leqslant j_{1}-1}\left\{X_{i}>a\right\}, \bigcap_{j_{1}+1 \leqslant i \leqslant j_{2}-1}\left(\left\{X_{i}<X_{j_{1}}\right\} \bigcup\left\{X_{i}>a\right\}\right),\right. \\
& \ldots, \bigcap_{j_{n-1}+1 \leqslant i \leqslant j_{n}-1}\left(\left\{X_{i}>X_{j_{n-1}}\right\} \bigcup\left\{X_{i}>a\right\}\right), \\
& \quad \bigcap_{j_{n-1}+1 \leqslant i \leqslant j_{n}-1}\left(\left\{X_{i}>X_{j_{n}}\right\} \bigcup\left\{X_{i}>a\right\}\right), \\
& \quad(1-F(a))^{j_{1}-1} \int_{\gamma}^{a}\left(1-F(a)+F\left(x_{1}\right)\right)^{j_{2}-j_{1}-1} d F\left(x_{1}\right) \\
& \quad \times \int_{x_{1}}^{a}\left(1-F(a)+F\left(x_{2}\right)\right)^{j_{3}-j_{2}-1} d F\left(x_{2}\right) \\
& \quad \cdots \int_{x_{n}-2}^{a}\left(1-F(a)+F\left(x_{n-1}\right)\right)^{j_{n}-j_{n-1}-1} d F\left(x_{n}-1\right) .
\end{aligned}
$$

Из последней формулы и получаем требуемое.

Утверждение 3.3. $\mathrm{E} T^{a}(n)=\infty(n \geqslant 2)$.

Д о к а з а т е л ь с т в о. Доказательство можно получить непосредственно из формулы (3.2), однако мы воспользуемся более изяцным методом, предложенным рецензентом.

Если из последовательности $X$-ов выбросить все те $X_{i}$, значения которых принадлежат интервалу $(a, \beta)$, то распределения величин $V^{a}(1), V^{a}(2), \ldots$ не изменятся. Среди оставшихся $X$-ов величины $V^{a}(i)$ будут уже классическими рекордами. Поэтому, вместо величин $X_{1}, X_{2}, \ldots$ с функцией распределения $F$ можно рассматривать новые величины $Z_{1}, Z_{2}, \ldots$ с функцией распределения $G(x)=F(x) / F(a), \gamma \leqslant x \leqslant a$, и тогда $\left\{V^{a}(k)\right\}_{k=1}^{\infty} \stackrel{d}{=}\left\{Z_{(k)}\right\}_{k=1}^{\infty}$, где $Z_{(k)}-$ уже обычные рекордные величины в последовательности $Z$-ов, и, кроме того,

$$
T^{a}(n)=L(n)+\mu(L(n))
$$

где $L(n)$ есть номер $n$-й рекордной величины в последовательности $Z$-ов, а $\mu(n)-$ общее число $X$-ов, отброшенных до появления $Z_{(n)}$. Отсюда сразу следует, что $\mathbf{E} T^{a}(n) \geqslant \mathbf{E} L(n)=\infty(n \geqslant 2)$.

Утверждение 3.4. Pаспределение $n$-й величины «o.n.n.» при $n \geqslant 2$ задается формулой

$$
\mathbf{P}\left\{V_{(n)}^{a}<x\right\}=\frac{1}{(n-1) !} \int_{0}^{-\log (1-F(x) / F(a))} \nu^{n-1} e^{-\nu} d \nu .
$$

Д о к а з а т е ль с т в о. Доказательство следует из известной формулы для распределения $n$-й рекордной величины, когда вместо $F(x)$ берется функция $G(x)$. 
Утверждение 3.5. Производящая фуякчия величины $T^{a}(n)$ имеет вид

$$
P_{n}(s)=\mathrm{Es}^{T^{a}(n)}=P_{L(n)}(s K(s)),
$$

2de

$$
K(s)=\frac{F(a)}{1-(1-F(a)) s}, \quad P_{L(n)}(u)=\frac{1}{(n-1) !} \int_{0}^{-\log (1-u)} \nu^{n-1} e^{-\nu} d \nu .
$$

Последняя функция является производячей Функчией рекордных моментов (результат Реньи [1], 1962).

Д о к а з а т е л ь с т в о. Рассмотрим схему Бернулли с вероятностью «успеха» $p=\mathbf{P}\{X \in(\gamma, a)\}$ и вероятностью «неудачи» $q=1-p=\mathbf{P}\{X \notin(\gamma, a)\}$. В этом случае число неудач (среди величин $X_{i}$ ) до появления $(L(n)=m$ )-ro успеха имеет отрицательное биномиальное распределение с параметрами $p$ и $m$. Справедлива формула

$$
\mathbf{P}\left\{\left\langle T^{a}(n)=k+m \mid L(n)=m\right\rangle\right\}=C_{m+k-1}^{k} F^{m}(a)(1-F(a))^{k} .
$$

Производяшая функция рекордных моментов имеет вид (см. [1]):

$$
P_{L(n)}(s)=\mathrm{E}^{L(n)}=\frac{1}{(n-1) !} \int_{0}^{-\log (1-s)} \nu^{n-1} e^{-\nu} d \nu .
$$

Откуда

$$
\begin{aligned}
P_{n}(s) & =\mathbf{E} s^{T^{a}(n)}=\mathbf{E}\left\{\mathbf{E} s^{L(n)+\mu(L(n))} \mid L(n)\right\} \\
& =\mathbf{E}(s K(s)) \stackrel{L(n)}{=} P_{L(n)}(s K(s)) .
\end{aligned}
$$

Формула (3.4) позволяет также находить условные моменты. Так, например, $\mathbf{E}\left\{T^{a}(n) \mid L(n)=m\right\}=m / F(a)$, откуда

$$
\mathbf{E}\left\{\frac{T^{a}(n)}{L(n)}\right\}=\frac{1}{F(a)}
$$

Автор работы выражает глубокую признательность рецензенту за предложенные в рецензии методы. Эти подходы позволили сократить доказательства и обогатить содержание работы. Так, в новой редакции статьи более половины всех результатов получено из критических замечаний рецензента.

\section{СПИСОК ЛИТЕРАТУРЫ}

1. Renyi $A$. On the extreme elements of observations. - In: Selected papers of Alfred Renyi. V. 3. Budapest: Akademiai Kiado, 1976, p. 50-65.

2. Галамбош Я. Асимптотическая теория экстремальных порядковых статистик. M.: Наука, 1984, 304 c.

Поступила в редакцию 29.IX.1994 